\section{Panorama de las politicas públicas de seguridad ciudadana en Cartagena}

View of the public policies of public safety in Cartagena

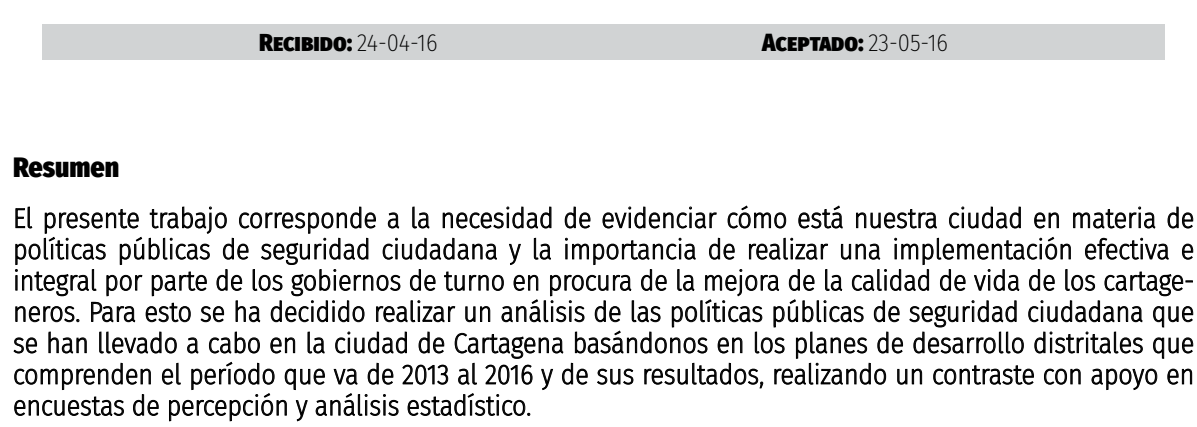

Palabras clave: políticas públicas, seguridad ciudadana, calidad de vida, efectividad, Cartagena

\section{Abstract}

The present work corresponds to the need to demonstrate how our city is in public policy issues of citizen securty and the importance of implementing an effective and integral implementation by the governments
of these in order to

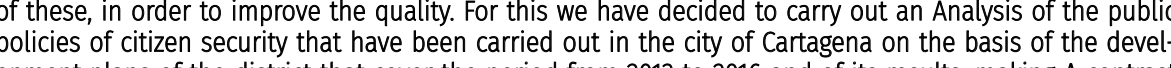
pment plans of the district that cover the period from 2013 to 2016 and of its results, making A contrast

Keywords: public policies, citizen security, quality of life, efficiency, Cartagena.

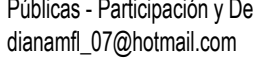

2 Estudiante de VII semestre de Derecho de la Universidad de Cartagena, perteneciente al Semillero de Invesigación. Politicas Públicas - Participación y Desarrollo.
yulissajaimespernett@outlook.com
La seguridad ciudadana en Cartagena se ha convertido en tema de gran discusión y exige atención de urgencia por sus graves dimensiones debido a la aparente falta de esta en la ciudad, mientras que los gobiernos locales plantean soluciones a corto plazo, sectorizadas e insuficientes, los ciudadanos sienten que están siendo categorizados y excluidos con las políticas públicas Además con el cambio de perspectiva que se ha ido generando a nivel internacional y nacional en cuanto al papel e importancia de la seguridad ciudadana $y$ al giro en su enfoque y tratamiento, en el que se combinan medidas no solo de control, sino de prevención con la inclusión de la participación de las comunidades, se hace necesario analizar cómo la ciudad está abordando el tema, cuál es el enfoque dado y la implementación de políticas que le den un tratamiento integral y efectivo a los fenómenos de criminalidad, todo esto con el fin de mejorar la calidad de vida de los cartageneros.

A continuación se realizará un análisis sobre el concepto de seguridad ciudadana, los parámetros necesarios para que una política pública sea efectiva, en especial una de seguridad ciudadana que será el enfoque y evidenciar cuál es el panorama que se afronta en esta materia en la ciudad de Cartagena; se realizará un análisis respecto a las políticas y acciones aplicadas en la ciudad y el contraste frente a los resultados y percepciones; finalizaremos planteando una serie de conclusiones a manera de reflexión.

\section{JUSTIFICACIÓN}

Las políticas públicas implementadas en un territorio determinado deben tener un papel real en los efectos que desean buscar; deben verse reflejadas en resultados altamente efectivos de tal forma que cumplan con el fin último de su acepción como lo es la garantía y protección de uno o más derechos y la satisfacción de las necesidades en los habitantes. Sin embargo, resulta compleja esta garantía si se tiene en cuenta que las necesidades de los ciudadanos cartageneros en materia de seguridad se han visto afectadas en gran manera por factores alternos; aqui entramos a cuestionarnos, ¿existen en la ciudad de Cartagena políticas públicas altamente efectivas y eficaces que combatan la inseguridad ciudadana y promuevan una convivencia pacíica en los cartageneros?, esto se verá reflejado en la percepción que los habitantes tienen respecto a este tema. Es por esto que se pretende con la investigación estudiar los mecanismos y lineamientos que las políticas públicas 
de seguridad ciudadana deben cumplir al ser estructuradas, de manera que tengan una alta efectividad en los resultados que buscan.

\section{METODOLOGÍA}

El presente trabajo de investigación es realizado de manera jurídico-conceptual mediante un desarrollo analítico y documental, diagnóstico-descriptivo, basado en fuentes primarias y secundarias como son textos científicos, encuestas, estadísticas y la normativa referente.

\section{CONCEPTOS GENERALES}

\section{Seguridad ciudadana}

La seguridad ciudadana abarca el desarrollo de conceptos de delincuencia, situaciones de vulnerabilidad y riesgo para personas y sus bienes y la policía pública como ente encargado de proteger lo vulnerado; a partir de esta se encuentra que la seguridad ciudadana supone la existencia de niveles sociales inconformes con el hecho de la desprotección estatal, aquí es donde entra el estado como protector y velador de los derechos de las personas a cumplir un papel fundante como lo es la protección y el bienestar de sus asociados, en donde debe existir un acompañamiento por parte de la ciudadanía mediante una colaboración y representación de sus intereses. Se resalta que:

La seguridad ciudadana puede definirse como una necesidad social. Este concepto se refiere a las exigencias especíicas de la población vinculadas con la delincuencia y las situaciones de vulnerabilidad y riesgo para sus personas y bienes, las cuales estarían estrechamente asociadas a la policía pública, que tiene la función de resolver, o al menos minimizar, los efectos negativos de dichas amenazas. (Monsalve, 2009)

En la actualidad la seguridad ciudadana es el resultado de un cambio social con un miedo generalizado y la espera de prontas soluciones por parte del Estado, por lo que requiere un cambio paralelo de las acciones estatales y gubernamentales que de manera eficaz y exitosa confronten los problemas actuales en materia de seguridad ciudadana con el acompañamiento ciudadano y bajo la protección de los intereses y bienes de este. Se ha dicho que se ha instaurado la seguridad ciudadana como la representación de un interés colectivo; el acceso a una vida segura, es sin duda, el mayor logro de quienes hoy ejercen el control del escenario político (Candia, 2002).

La idea de seguridad ciudadana supone aunado a la democracia, un régimen político donde hay participación y representación de los intereses de la población en el proceso. En el punto del acompañamiento policial puede entenderse como el fortalecimiento de las instituciones del Estado que procuran por la satisfacción de dicho bienestar y por el orden social como base de la convivencia, donde debe existir una delimitación entre la importancia o relevancia de dichas instituciones y el derecho mismo de las personas, es decir, no se debe olvidar que el fin último de la actuación administrativa debe ser el ciudadano.

\section{Políticas públicas}

Con la entrada del siglo XX, la globalización y la creación de redes estratégicas entre los países para la reducción de índices desfavorables estatales, el crecimiento económico mundial y la apertura económica han retado a los Estados al diseño de nuevas estrategias y políticas públicas en las que se vean reflejados los esfuerzos y el progreso nacional, más allá del enfoque en temas de seguridad, defensa y todo lo relacionado con el área militar. A su vez las nuevas generaciones proponen problemas distintos que resultan en $\mathrm{el}$ surgimiento de nuevos planes institucionales.

De acuerdo a lo mencionado, una política pública es el resultado de la actividad de una autoridad investida de poder público y de legitimidad gubernamental (Meny y Thoenig, 1992). Bajo este entendido la política pública es el eje principal de un gobierno, dividido en distintas aristas que van encaminadas a la solución de problemáticas previamente identificadas bajo el fin de la estabilidad de cada territorio o nación, acompañadas de planes de acción que buscan el cumplimiento de las mismas mediante operaciones adminis trativas realmente eficaces, todo esto bajo el marco legal y jurídico en procura del orden público.

\section{PARÁMETROS PARA LA CREACIÓN DE POLÍTICAS PÚBLICAS EFICACES}

Para el desarrollo de las políticas públicas dentro del plano institucional y su eficacia social se han propuesto tres enfoques de los distintos modelos teóricos 
que plantean un verdadero cumplimento de las mismas. Se desarrollan desde tres puntos de vista:

El primero, centrado en el enfoque <social>, privilegia al individuo y el pluralismo social y solo concibe el Estado, desde una perspectiva funcionalista, como una ventanilla encargada de atender las demandas sociales.

Un segundo grupo de teorías, al contrario, insiste en atribuir al Estado la condición de instrumento al servicio de una clase o de grupos específicos. Según esta óptica, el Estado dispone sólo de una autonomía marginal, ya represente los intereses del capital (teorías neomarxistas) o de los burócratas o expertos que lo controlan desde su interior (teorias neoweberianas).

Finalmente, un tercer conjunto intenta encontrar un camino intermedio, dedicándose a interpretar los equilibrios y desequilibrios que se establecen entre el Estado y la sociedad y que las políticas públicas permiten traducir. (Meny y Thoenig, 1992)

Tomando el último enfoque como el punto intermedio e ideal de la creación, formación y desarrollo de toda política pública en el que se propende por un equilibrio social más que por el interés particular, y para el verdadero y eficaz cumplimiento y realización. Se ha mencionado que

Para asegurar el éxito de las políticas de seguridad ciudadana deben considerarse tres elementos claves: Información, Gestión integral y Cooperación para el conocimiento, por lo cual es ideal precisar ciertas características que deben ser analizadas para la creación de estas que desenvuelven lo anterior. (Frühling, 2012)

De aqui pueden desprenderse los siguientes elementos:

\section{Información verídica y rigurosa que permita identificar el problema} que se va a intervenir: se necesita una verdadera individualización del tema a tratar, su contexto, las situaciones que lo genera, el desarrollo de esta situación a lo largo de los años, los sujetos que inciden en ella y las soluciones que se han planteado previamente, mediante estudios de monitoreo cualitativos y cuantitativos de índices económicos, políticos, sociales, culturales y de percepción ciudadana en torno a dicho problema a tratar, a esto se le conoce como información estadistica de calidad. Frühling (2012), menciona que es necesario e importante para que los proyectos tengan real efectividad que exista información estadística de calidad que permita priorizar las falencias y facilite el seguimiento de los avances y las intervenciones en $\mathrm{si}$.

\section{Estudio detallado de evaluación e identificación de puntos de inflexión}

de políticas públicas anteriores con resultados fracasados y que permita deducir el éxito de las siguientes: una vez identificado el problema a intervenir deben tenerse en cuenta dos posibles resultados para las políticas públicas implementadas: el éxito (total o parcial) o el fracaso. Este punto se divide en dos estudios: por un lado se observan los puntos de inflexión que conllevan al fracaso de una política pública y en los que se busca el análisis detallado de políticas públicas previas desarrolladas para los mismos puntos con el fin de localizar los puntos negativos y desviados de cada esquema organizacional y su desarrollo, con el fin de neutralizar y crear estrategias de prevención, un mayor sistema organizativo y mejores planes de gobierno.

Y por otro lado la construcción de nuevos esquemas institucionales que se vean determinados por verdaderos estudios de aplicación, en los que se tengan en cuenta acciones como la evaluación de impacto de las políticas públicas en las principales ciudades a nivel mundial para la observación de cumplimiento de dichas políticas públicas en su territorio, es decir, la efectividad y eficacia que lograron y los niveles de disminución del problema que se planteaba a solucionar y la contextualización de estas dentro del territorio a aplicar, con base en la pregunta ¿qué tan eficiente resulta la aplicación de " $X$ " política pública conforme a los parámetros y estándares sociales, culturales, económicos y políticos del espacio de aplicación? Al analizar los resultados de estudios respecto al tema se hace alusión a que, la única alternativa posible para determinar si alguna iniciativa funciona en nuestro contexto es testearla de acuerdo con métodos rigurosos (Frühling, 2012), generando una relación causal entre el punto primero cuando se refiere al estudio estadístico de las situaciones territoriales y el aquí detallado.

3. Efectividad y preparación en la acción gubernamental: en el entendido que toda política pública va acompañada de las operaciones administrativas por las cuales se lleva a cabo el plan de desarrollo estipulado, la acción gubernamental debe estar totalmente profesionalizada y capacitada mediante creación de talleres, conferencias y capacitaciones de los empleados públicos con respecto a la labor que se encuentran desarrollando, una concientización 
del respeto y eficiencia con que se deben llevar a cabo dichas operaciones en calidad de prestación de un servicio a la comunidad propendiendo el bien general y el seguimiento e investigación de aquellos que en función de su labor pública realicen conductas inapropiadas o negligentes que estanquen y perturben los procesos. Por otro lado es necesario el estímulo de un trabajo interadministrativo conjunto de las instituciones estatales en procura del buen desarrollo operacional, identificando principalmente cuáles problemas se encuentran en conexión bajo los temas de enfoques sociales, económicos, culturales y políticos ya mencionados, a esto se le conoce como prevención social; en últimas, reconocer qué instituciones en alianza y trabajo mancomunado necesitan la estructuración de planes que soporten las políticas públicas a desarrollar. En el estudio temático se resalta que estas requieren de un aumento en la capacidad estatal para llevar a cabo intervenciones multisectoriales que parecen prometedoras en la región y que coordinan la actuación de diversos organismos públicos situados a nivel central, regional y local (Frühling, 2012).

Todo lo anterior bajo las instancias de políticas públicas ligadas al estudio, aplicación y vigencia del derecho, entendiendo lo anterior como la acción conforme a derecho que deben realizar las instituciones y el control de los funcionarios de acuerdo a un régimen sancionatorio disciplinario para garantizar el normal y adecuado funcionamiento y la eficiente y transparente actividad operacional.

\section{Desarrollo concomitante de políticas públicas encaminadas a la mejora} de situaciones alternas que afecten directamente la política a establecer:

las políticas públicas como objeto de desarrollo y mejoramiento de situaciones circunstanciales que buscan reparar una afectación, promover el crecimiento de determinado punto o mantener ciertos estándares y resultados positivos, debe trabajarse de la mano con las políticas alternas, la complementariedad de estas determinará en gran parte la efectividad de las mismas. Apoyados en Frühling, Tulchin y Golding (2005), se precisa que otro obstáculo que enfrentan las reformas es la tensión entre las soluciones a corto plazo para los problemas inmediatos y la necesidad de atacar las causas profundas del problema, estrategia que solo puede producir resultados a largo plazo. Esto considera que la existencia de políticas públicas que satisfagan las causas principales de la problemática planteada inicialmente servirán de soporte para la efectividad de la misma, considerando en ejemplo las políticas públicas de empleo no podrían ser satisfechas sin unas políticas públicas educativas sostenibles.

5. Percepción y resultados de las políticas públicas. Mejoramiento de las directrices: en último análisis es indispensable verificar los resultados de toda política pública, el éxito de su desarrollo y la eficacia y permanencia de estos en el transcurso temporal, si lo que se busca como fin último es la solución de un problema ya identificado y se cumple con los parámetros propuestos dentro de un marco general contextual, se debe evaluar la percepción y los indices que reflejan los efectos, entran de nuevo los cuadros y estudios estadísticos, para la evaluación y diagnóstico de mejoras y medición de resultados generales, en los que se necesita, al igual que los puntos anteriores, una gran inversión tanto económica como profesional, capacitada y completa.

De manera enfática se presenta otra serie de necesidades con respecto al tema de seguridad ciudadana para que la proposición de políticas públicas genere programas gubernamentales eficaces y dé resultados notables, perceptiva y fisicamente, como lo son:

- Visión igualitaria de la problemática social: las políticas públicas deben estar encaminadas a solucionar situaciones sociales que a pesar de verse focalizada en lugares determinados de la población y el territorio, generan afectaciones generales, por lo que debe replantearse la concepción excluyente de estas y trabajar por la satisfacción armónica de las necesidades. En la ciudad de Cartagena el Decreto 1424 (2016) basa su estructura en lo que la misma menciona como la metástasis que ha orientado el accionar de bandas de delincuencia y en algunos casos estructuras de crimen organizado con respecto a los sectores de la ciudad mencionados (par. 8), de los cuales se puede evidenciar conforme al Decreto 0977 (2001) se encuentran como estratos predominantes (Art. 118). Evidenciando la exclusión del resto de barrios y sectores de la ciudad, en los que la seguridad ciudadana se limita a políticas excluyentes y sectorizadas. Se menciona que

Hay que buscar políticas de seguridad inclusivas que protejan a los diversos actores y no solo los derechos de un determinado secto social, y mucho menos que promuevan la seguridad de un determinado grupo social a costa de los derechos de los demás ciudadanos. (CELS y otros, 2004) 
- Moderación de las políticas represivas y trabajo social reforzado: el proceso de crecimiento del país ha sembrado en la ciudadanía una cultura de miedo, lo que se ve reflejado en la incapacidad de la población de enfrentar situaciones de inseguridad debido a las repercusiones que estas puedan acarrear, dando paso a una cultura represiva donde los derechos y libertades se veían cohibidas más que respetados y dados en cumplimiento. Las políticas públicas de seguridad ciudadana deben estar focalizadas hacia el respeto de los derechos fundamentales de los habitantes y la visibilización de estos en la sociedad. Otro punto importante lo representa el trabajo, no basta el fortalecimiento de las instituciones de coerción si no existe un trabajo mancomunado con la población, una identificación de situaciones generadoras de problemas y la búsqueda de la satisfacción de las mismas, al igual que un constante seguimiento del desarrollo de la gestión.

\section{- Identificación y particularización de situaciones reales causantes del} problema de seguridad ciudadana: la mentalidad estatal y ciudadana de la delincuencia como una fuerza que debe ser atacada por una fuerza mayor debe ser revaluada, no puede tomarse en cuenta como un fenómeno aparte e individual, las políticas públicas deben estar interrelacionadas con políticas sociales tendientes a disminuir factores de riesgo y situaciones generadoras de miseria que representan en último sentido la raíz del problema de inseguridad; factores como la desigualdad y la exclusión social en temas laborales, educativos, de salud y culturales, repercuten en los sectores menos favorecidos de la sociedad. Si bien, la identificación de situaciones reales y su propugnación por la mejora en seguridad ciudadana refleja una necesidad de mejora en políticas penales y judiciales; también deben ser reestructuradas políticas de empleo para jóvenes, políticas inclusivas de salud donde la cobertura de este sistema sea mayor y políticas educativas encaminadas a la primera infancia y los primeros niveles, debe promoverse que al igual que la efectividad de los programas de seguridad ciudadana este debe ser tenido en cuenta; en una cadena interdependiente es necesario que como pilar de un programa de gobierno cada política pública genere los resultados previstos; este punto debe ver reflejado la inclusión social y participación real de los habitantes; de manera conjunta la inclusión de los habitantes de cada sector debe verse reflejada en grupos de convivencia de seguridad ciudadana, debe existir una implementación mediante campañas pedagógicas y actividades.
III. LA SEGURIDAD CIUDADANA COMO POLITICA PÚBLICA

El temor a la inseguridad se ha convertido en un aspecto de especial significancia como parte de la incertidumbre y de la crisis producida por los cambios de orden político, económico, social y cultural de nuestro mundo globalizado, este siempre ha existido en todas las épocas, pero hoy se ha convertido en el foco principal de los grandes debates a nivel mundial, debido al miedo generalizado que existe actualmente.

El aumento de la violencia y la inseguridad en nuestras ciudades ha llevado a sus habitantes a desarrollar reflejos de defensa, como la contratación de guardias privados, la instalación de alarmas, la construcción de muros para autoclausurarse en sus propios domicilios y, en casos extremos, la justicia po mano propia. Esos comportamientos ciudadanos evidencian que el Estado ha perdido su condición de garante de la paz. Se ha roto la figura del contrato social en la que los individuos se asocian en comunidad y entregan al Estado la autoridad para velar por el interés colectivo.

La confianza ha desaparecido y en su lugar se ha instalado el miedo, la emoción más atentatoria contra la capacidad de raciocinio, y el estado emocional que determina actualmente la actitud de las personas frente a su entorno y a sus semejantes. (Marchan, 2004)

A partir del enfoque de seguridad ciudadana surge entonces la arista de seguridad nacional basada en el Estado como garantizador de la estabilidad nacional en donde la seguridad de los ciudadanos se ve intrínsecamente ligada a la del régimen político y a la defensa de los intereses nacionales. Conforme a este contexto existen dos elementos constitutivos de la seguridad:

El primero es la importancia del Estado como garante de la seguridad de la población. De igual manera, se percibe a este como una forma de evolución, y se ve en él la máxima expresión política de la sociedad. En segundo lugar, se puede apreciar un enfoque esencialista o minimalista de la seguridad, es decir, que esta tiene que ver estrictamente con la supervivencia y no con el bienestar. (Jaramillo, 2015)

La realidad latinoamericana en este sentido plantea cambios en la visión tradicional de seguridad desde una dejación de la perspectiva militar de la seguridad y una conversión de este concepto en la satisfacción de normas 
reales efectivas que garanticen el buen funcionamiento de los planes de desarrollo, así como la buena realización de estos mismos conforme a las dinámicas constructivas latinoamericanas con respecto a seguridad ciudadana.

Una vez empieza a emerger una crisis de seguridad ciudadana como realidad en cada sociedad, producida por procesos de urbanización anteriores que trajeron consigo el hecho de una insostenibilidad de las urbes y sus gobiernos en complacencia de la sostenibilidad de sus habitantes debido al abrupto crecimiento, así pues esta carencia de entes y acciones gubernamentales no suscitan solo en necesidades básicas sino que producto de esto también surge una insatisfacción al sistema para confrontar temas como la inseguridad que desborda en las ciudades, obligando a cada gobierno en sí mismo ana reestructuración de sus políticas, planes y actuaciones para el abarcamiento de todo lo que conlleva la seguridad ciudadana.

Asi entonces las actuaciones deben estar generalizadas, en el sentido que deben proteger más que intereses de turno, los intereses de la sociedad como colectivo y de los ciudadanos en particular, por lo que se requiere por parte del Estado una participación activa de elaboración de políticas públicas de seguridad ciudadana además de militarizadas y con una orientación controladora y reactiva, un enfoque mucho más preventivo, donde más allá de un fortalecimiento de las instituciones penales se requiere de acciones reales encaminadas a evitar las raíces generadoras de crimen y violencia para el mantenimiento general del orden público y en últimas la seguridad ciudadana

Alternativa, cuya iniciativa y liderazgo se centra en las comunidades locales y sus organizaciones y que por vía de la prevención trata de establecer una línea de acción pública que a través de medidas alternativas y alternativas no penales, oriente su actividad hacia la resolución de los conflictos sociales sin necesidad de recurrir a la criminalización. (López \& García, 2001).

En orden de ideas, las políticas públicas de seguridad ciudadana deben responder a las necesidades colectivas raíces de las situaciones generadoras, es decir, deben atacarse 0 satisfacerse los problemas principales que conllevan a que el ciudadano de menos recursos proceda a los métodos delincuenciales como manera de subsistir. Las políticas públicas en materia de seguridad ciudadana deberán garantizar la dignidad humana y calidad de vida de los habitantes del territorio, con sistemas integrales e integradores de las necesidades, soluciones y los ciudadanos como ente principal en esta cadena. En este sentido ya expuesto y de manera sintetizadora del tema, bien

se ha dicho que

Una política pública de seguridad ciudadana será un conjunto organizado y estructurado de acciones, que buscan generar situaciones, bienes y servicios públicos, para satisfacer las demandas de los ciudadanos, transformar condiciones de vida, modificar comportamientos, generar valores 0 actitudes que correspondan con la ley, la moral y la cultura propios de una comunidad. De acuerdo con lo anterior, es coherente precisar que la seguridad es un bien que apunta a la calidad integral de vida de los ciudadanos y no solo a la disminución o ausencia de delitos y hechos de violencia. (Policía Nacional, 2010)

\section{SEGURIDAD CIUDADANA EN COLOMBIA}

En Colombia los periodos de violencia y terror no han sido fenómenos nuevos, caracterizándose por su complejidad y multidimensionalidad han marcado el proceso de construcción de las bases de nuestro Estado, la crisis de seguridad durante los años 40 y 50 conocida como el periodo de la violencia desencadenado por la guerra civil entre los dos partidos tradicionales, conservadores y liberales, son un ejemplo de esto, así como las posteriores crisis de los años $60,70,80$, llegando a su máxima expresión en los 90.

La crisis que experimentó Colombia entre los años 60 a los 90 , se debió a varios factores, como el surgimiento de nuevos fenómenos de violencia y criminalidad con el nacimiento de las guerrillas, grupos de terrorismo y crimen organizado; posteriores al periodo de violencia, el vacío de una política de Estado enfocada en la prevención y en la garantía a la seguridad ciudadana, los altos niveles de impunidad y corrupción de la justicia y la creencia por parte de alcaldes y gobernadores de que el asunto de seguridad era de competencia exclusiva del Gobierno Nacional, debido a la falta de descentralización del manejo de la seguridad en el país.

La década de 1990 se destaca como una época de cambio e innovación en el manejo del crimen y la violencia, cambios que se remiten a la redefinición del campo de la seguridad con la inclusión en la Constitución de 1991 de los conceptos de seguridad ciudadana y convivencia pacífica y la delimitación que 
en esta se da de las competencias y directrices para su manejo en manos de las autoridades locales como primeras autoridades de policía, aspecto esencial en el florecimiento de iniciativas locales orientadas al manejo del crimen y la violencia, además de la contribución de las iniciativas presidenciales en seguridad sancionadas a lo largo de la última década en las que es posible hablar de una tendencia común, ya que estas aunque de formas diferentes y en ocasiones casi opuestas, incorporan la idea de una respuesta integral; así como la referencia a temas relativos al conflicto armado y el narcotráfico a la par con la defensa de los derechos humanos, el mejoramiento de la justicia, y la participación activa de los ciudadanos y su capacidad de relacionarse de manera no violenta, como eje de la generación de seguridad y la lucha contra el crimen y la violencia (Rivas, 2003).

En el complejo contexto de la seguridad en Colombia en el que por una parte se encuentra la necesidad de contrarrestar las acciones de grupos armados y de actos terroristas y por otro lado mitigar el miedo a la inseguridad en las ciudades, se combinan la seguridad pública y ciudadana en acciones con enfoques y tecnologías que puedan abordar de manera más sistemática e integral la seguridad, aplicando y reconociendo la necesidad de armonizar iniciativas de mano dura o convencionales, de control y de mano blanda que se enmarcan en la prevención de la violencia.

El marco de las políticas públicas de seguridad ciudadana a nivel nacional se encuentra en el Plan Nacional de Desarrollo y en la política nacional que se fije en esta materia; para efectos de nuestro trabajo nos apoyaremos en en Ley 1450 de 2011 y en la Ley 1753 de 2015. En cuanto a la primera, se estableció la consolidación de la paz; el logro de mayores niveles de seguridad, el funcionamiento eficaz de la justicia y como uno de los componentes claves para la consolidación de la seguridad y el orden público nacional, la seguridad ciudadana; señalando la necesidad de una política nacional de seguridad y convivencia ciudadana que de una manera integral, coordinada, permanente y a largo plazo pudiera atender reconociendo las peculiaridades de cada territorio a través de respuestas que incluyan acciones de prevención, control y aplicación de justicia penal, resocialización y asistencia a las víctimas del delito, las múltiples expresiones delictivas en los territorios (Ley 1450, 2011).

Por otra parte el diagnóstico en materia de seguridad ciudadana dentro de la Ley 1753 de (2015) señala que aunque el país ha avanzado en desarrollo de instrumentos de planeación local y líneas de acción específicas en esta materia debido a que en la actualidad el país cuenta con 836 planes integrales de seguridad y convivencia ciudadana municipales (equivalentes al $74 \%$ de los municipios del país) y 32 departamentales (enmarcados en los lineamientos fijados por la Política de Seguridad y Convivencia Ciudadana), aún existen retos asociados con:1) ausencia de estrategias específicas en las áreas rurales; 2 ) inexistencia de un sistema que articule la información relacionada con el delito; y, 3) uso ineficiente de las herramientas tecnológicas para la seguridad ciudadana.

Este plan de desarrollo trabaja sobre dos objetivos para alcanzar la paz, los cuales son el fortalecimiento del rol del Estado para garantizar el goce efectivo de los derechos de los colombianos en sus territorios proveyendo más seguridad, justicia y democracia y el segundo enmarcado dentro de la superación del conflicto armado, la Justicia Transicional y el derecho de las víctimas; dentro del primer objetivo para proveer seguridad y defensa en el territorio nacional se manifiesta la necesidad de complementar la seguridad y convivencia ciudadana junto a la seguridad pública y la soberanía nacional para la construcción de la paz dentro de un proceso de posconflicto en el cual uno de los principales retos será la seguridad y convivencia ciudadana a través de las líneas de acción, prevención social y situacional del delito. Lucha contra las principales modalidades delictivas que afectan a los ciudadanos, implementación de tecnologías para la seguridad ciudadana, fortalecimiento de la presencia policial, Centros de Integración Ciudadana (CIC), Sistema Nacional de Información y Análisis del Delito (SNIAD).

Dentro del enfoque del Plan Nacional de Desarrollo en la construcción de la paz y el posconflicto se conectará la política de defensa y de seguridad 2015-2018, la cual tendrá como uno de sus objetivos estratégicos, garantizar mayores y mejores niveles de seguridad ciudadana fortaleciendo las relaciones del ciudadano con el Policía, las cuales abordarán las mismas líneas estratégicas del Plan Nacional de Desarrollo.

\section{ANÁLISIS DE LAS POLITICAS PÚBLICAS DE SEGURIDAD CIUDADANA E}

Dentro del proceso de descentralización establece la Constitución Política de Colombia (1991) una jerarquía en el manejo del orden público atribuyendo funciones y responsabilidades en materia de seguridad ciudadana tanto 
gobernadores como a alcaldes. En desarrollo de los preceptos constitucionales mencionados, se establece también que alcaldes y gobernadores son las primeras autoridades de policía a nivel departamental y municipal respectivamente (Ley 62, 1993). Las directrices nacionales en el marco de las políticas públicas de seguridad ciudadana establecen los lineamientos que se deben seguir en el desarrollo de los instrumentos de planeación territorial; los planes de desarrollo territoriales y los planes integrales de seguridad y convivencia ciudadana.

Partiendo de los parámetros de creación e implementación de políticas públicas descritos con anterioridad y las funciones atribuidas legalmente a las autoridades locales, es preciso realizar un análisis de la aplicación de estos en las políticas públicas de seguridad ciudadana en la ciudad de Cartagena para el periodo de mandato actual, sin embargo de manera sucinta cabe hacer un análisis del programa de gobierno perteneciente al exalcalde Dionisio Vélez Trujillo para el periodo 2013-2015 esbozado en el Acuerdo Distrital 016 de (2013) en el que se evidencia la estructuración de este con programas enfocados en el fortalecimiento de las instituciones encargadas del desarrollo en materia de seguridad ciudadana y encaminados al aumento de la capacidad administrativa, verbigracia, el programa de fortalecimiento institucional de la Secretaría del Interior, el fortalecimiento del sistema penitenciario, el fortalecimiento del Cuerpo de Bomberos y el fortalecimiento del sistema penal para adolescentes en la ciudad; de igual forma se describe el aumento de los programas tecnológicos, puntualizando en programas como la implementación del sistema de circuito cerrado de cable en la ciudad para la filmación de hechos ocurridos en el espacio y las vías públicas y los sistemas de alarmas comunitarias, con el programa acupuntura para la seguridad se pretendió recuperar e intervenir 15 barrios de la ciudad con mayores índices de inseguridad y violencia a través del plan integral de seguridad ciudadana y la recuperación de entornos urbanos deteriorados, además se estableció un programa para la promoción de prácticas de convivencia y cultura ciudadana y el fortalecimiento logístico en aras a mejorar la capacidad de respuesta, la vigilancia comunitaria por cuadrante y el acercamiento con la comunidad de los organismos de seguridad.

Según el informe técnico de seguimiento y evaluación del Acuerdo No. 016 de (2013) realizado por la Secretaría de Planeación Distrital (2015) que tiene en cuenta a la hora del análisis de los resultados los criterios de evaluación basados en la tabla de calificación de resultados de eficacia establecida por el DNP3, este debía tener un porcentaje esperado de implementación de un $75 \%$ pero solo logró un $68,5 \%$, lo que permite concluir que las acciones del Gobierno Distrital lograron un nivel medio en la ejecución de los logros; en este se evidencia teniendo en cuenta los criterios de evaluación de eficacia fijados, que el componente estratégico de seguridad logró un nivel medio de implementación de sus programas con un $52,83 \%$, resaltándose que su programa acupuntura para la seguridad obtuvo un porcentaje de ejecución de un $78 \%$ alcanzando un nivel alto y el programa fortalecimiento de la inteligencia que pretendía el fortalecimiento institucional de las entidades competentes en materia de seguridad y orden público en el Distrito como: Secretaría del Interior y Convivencia Ciudadana, Cuerpo de Bomberos y Cárcel Distrital de Mujeres, un nivel bajo con un $33 \%$.

Según las estadísticas del programa Cartagena cómo vamos (2015), en su informe de calidad de vida, se llegó a la siguiente conclusión en materia de seguridad: el gran aumento en muertes violentas que experimenta la ciudad sigue siendo preocupante desde el 2010, a pesar que el año 2015 fue el menos violento con 21 homicidios menos en comparación al 2014 desde el 2012, la tasa de homicidios sigue siendo alta en comparación a otras ciudades; el homicidio bajo la modalidad de sicariato seguido de las muertes por accidentes de tránsito, son las circunstancias en las que estas muertes violentas más se generan; el sicariato tuvo una disminución importante en el 2015 teniendo en cuenta los últimos 10 años, lo alarmante es que esta modalidad desde hace una década está siendo reemplazada por la violencia interpersonal, en particular en este año en el cual la mayoría de muertes fueron producto de riñas comunes y entre pandillas, alertando a la ciudad al fomento y fortalecimiento de la convivencia ciudadana y a la motivación de la cultura de la resolución pacífica de conflictos.

Según COSED (2015) en su diagnóstico estadístico final de delitos de la ciudad de Cartagena se concluye lo siguiente:

- Se evidencia que a pesar de que hubo una disminución de un $7 \%$ en los homicidios en comparación al 2014, año que se consolidó con el mayor número dentro de los últimos 20 años, esta no es suficiente para estar por

3 La eficacaia se entiende como el grado de cumplimientit de las metas y objetivos a nivel de productos y ressultados. Cartilla Güa Metododógica para el Seguimiento y Evaluación a Polliticas Públicas 2014, p.15. DNP. Dirección de Seguimiento y Evaluación de
Politicas Publicas. 
debajo de la cifra alcanzada en años anteriores en los que el promedio oscilaba entre los 230 y 250 casos como máximo, incluso en el 2008 el número de homicidios fue de 171

- Los homicidios por violencia interpersonal superan en cantidad a los ocurridos bajo el contexto de delincuencia organizada, lo cual empieza a convertirse en un factor característico de la nueva tendencia homicida en la ciudad, en la que sobresale la intolerancia como principal rasgo distintivo. Por su parte los casos de delincuencia organizada siguen disminuyendo.

- La violencia intrafamiliar en el 2015 fue la segunda más alta dentro de un periodo de 8 años superada solo en el 2009, teniendo mayor incidencia su expresión de maltrato a la pareja en la cual $82 \%$ de las víctimas son mujeres.

- Una tendencia creciente a partir del año 2010 de los delitos sexuales, que sigue en el 2015

- Los hurtos han ido disminuyendo en un $5 \%$ en comparación con el 2014 pero sin embargo en la percepción de los cartageneros es la mayor fuente de inseguridad en un $70 \%$

- En la extorsión hubo una disminución de 11 casos frente al 2014 y frente a los casos presentados desde 2008-2013 siendo este último año el de mayor cantidad.

Por otro lado, el programa de gobierno "Primero la Gente 2016-2019" esbozado en el Acuerdo $\mathrm{N}^{\circ} 06$ de 2016, liderado por el alcalde mayor actual, Manuel Vicente García Duque, a pesar de no manejar cifras estadísticas actualizadas oficiales, en su organización de gobierno el numeral tercero de este, plantea los programas y estrategias de la propuesta gubernamental de mejoras ciudadanas, enfocada bajo la perspectiva de la inversión de terceros sobre la ciudad, es decir, que el plan de gobierno se busca llevar a cabo por medio de políticas públicas de manera que la calidad de vida y el crecimiento económico en la ciudad se incremente, lo cual a fin de cuentas atraerá, como ciudad turística por excelencia, la visita de muchos extranjeros nacionales e internacionales y por ende el gasto e inversión de particulares y empresas sobre el territorio municipal y sus proyectos.

Así bien, entre diversos temas concernientes a tema de interés local como el turismo, las políticas de empleo, los problemas de vivienda y salud y otros más, se resalta la estrategia denominada Seguridad Integral, la que desarrolla de igual forma temas concernientes al transporte público local, la cultura ciudadana como fuente de preservación de los bienes colectivos de la ciudad fortalecimiento de los programas encargados de velar por las finanzas locales y la seguridad ciudadana, partiendo este último de la base de la búsqueda de una ciudadanía "empoderada" de su ciudad en convivencia armónica y tolerante bajo el título "ciudadanía sin miedos" se plantean los siguientes programas a desarrollar: barrio seguro, unidad comunera segura, fortalecimiento institucional de la Secretaría del Interior y Convivencia Ciudadana, Justicia cercana al ciudadano, Cartagena por la convivencia, Primero los jóvenes, Optimización tecnológica a las herramientas de seguridad, Fortalecimiento logístico a los organismos de seguridad, Socorro y justicia en el Distrito, Ordenamiento seguridad de las playas del distrito de Cartagena y el fortalecimiento institucional de distriseguridad; si se hace un análisis con el anterior plan de desarrollo del exalcalde Dionisio Vélez Trujillo, se puede llegar a la conclusión que en materia de seguridad se desarrollaron los mismos programas, solo que en este nuevo plan las estrategias y los subprogramas son mayores y se encuentran más estructurados y adaptados a las nuevas realidades, además establece algo importante que no se tuvo en cuenta en el anterior Plan de Desarrollo Distrital y es la formulación y ejecución de un Plan Integral de Seguridad y Convivencia Ciudadana por parte de la Secretaria del Interio aspecto de suma urgencia para desarrollar en la ciudad un tratamiento más efectivo e integral a las expresiones delictivas.

Debido a que es muy reciente la implementación del Plan de Desarrollo no son muchas las fuentes ni la información en las que nos podemos apoyar para analizar cómo vamos en materia de seguridad entre el año 2016 e inicios de 2017. Según documento de la Alcaldía sobre las conclusiones a las que se llegaron en el acta final de audiencia pública de rendición de cuentas el día 15 de diciembre de 2016, el Alcalde precisó el enorme reto que tienen que cumplir en cuanto a la reducción de los índices de delincuencia, dijo que para esto se está trabajando de la mano de la Policía y la Armada, habló de las instalación de cámaras de vigilancia que están beneficiando a 194 barrios de la ciudad en puntos de más delincuencia según estudios de la Policía y la instalación de alarmas, presentando testimonios de la comunidad.

De la encuesta de percepción ciudadana realizada por Cartagena cómo vamos en (2016) en cuanto a la seguridad, la percepción de inseguridad en los barrios por parte de los ciudadanos se ubicó en un $32 \%$, aumentando en relación al 
periodo anterior 2013-2015 en el que estuvo en un $29 \%$, siendo los problemas más graves según los cartageneros los atracos y las pandillas; por otra parte un $39 \%$ de los cartageneros se sienten inseguros en la ciudad, disminuyendo en un $1 \%$ respecto al periodo anterior en donde en promedio se ubicó en un $40 \%$, esto lleva a concluir que los ciudadanos se sienten más inseguros en la ciudad que en su barrio. Por otro lado la victimización fue alta en relación al periodo anterior, ubicándose en un $24 \%$, la cultura de la denuncia de los delitos sigue demostrando ser un problema en la ciudad donde el 51 \% dijo no haber denunciado en relación a un $49 \%$ que dijo haberlo hecho, mejora la percepción de la lucha contra la impunidad en relación al año 2015 aún así se debe seguir trabajando ya que la percepción de impunidad en un 51 \% sigue siendo alta.

\section{CONCLUSIONES}

Las dificultades y retos que se han desarrollado a lo largo de este artículo en materia de seguridad ciudadana suponen avances en estos tiempos en el país y específicamente como corresponde al trabajo en sí en la ciudad de Cartagena, ya que como se puede vislumbrar en el apartado del análisis de las políticas públicas en la ciudad, estas han tenido un crecimiento en cuanto a sus dificultades; ya que las autoridades locales han asumido y se han empoderado del papel institucional que les compete en el manejo y control de la seguridad; se puede afirmar que se ha pasado de la letra muerta a la práctica en este sentido, por otra parte se deben destacar los esfuerzos que se han encaminado en esta materia al fortalecimiento institucional que han emprendido las autoridades locales a través de iniciativas para su modernización e innovación, como las reformas a la Policía Nacional y el manejo de información técnica y científica para el manejo del crimen y la violencia, además del desarrollo de herramientas que permitan contar con información adecuada para el diseño de las políticas de seguridad, se ha buscado el acercamiento, la participación y el trabajo mancomunado de la comunidad y los demás actores sociales (la administración distrital y los órganos policiales) en la construcción de las políticas públicas permitiendo la inclusión de temas que afectan de manera crucial la seguridad y con esto la calidad de vida de los cartageneros, asi también se han generado estructuras dentro del Gobierno Distrital que se centran exclusivamente en el manejo de la seguridad para la sostenibilidad de los procesos, aún se sigue avanzando en la incorporación de diagnósticos previos y evaluaciones periódicas, como elementos constitutivos en el manejo de la seguridad a nivel local y así como poco a poco se ha ido avanzando en el manejo de la seguridad más allá de un manejo meramente policial combinando estrategias de "mano blanda" y "mano dura".

A pesar de los avances aún queda mucho por hacer y superar en cuanto al suministro de información con la cual se evalúan los índices del delito esto $s$ evidencia en el informe de COSED (2015) que expresa la dificultad que se tiene para la obtención de la información que está en custodia de la entidades que tienen la información primaria; a esto se suma la ausencia de información que permita llegar a resultados más objetivos debido a la falta de la cultura de denuncia que aunque ha mejorado sigue siendo baja, además de casos que permanecen ocultos por el miedo de las víctimas a las represalias que puedan tomar sus agresores.

Por otra parte se encuentra la falta en la ciudad de una planificación seria en cuanto a políticas de seguridad ciudadana, es más, la ausencia de una política pública en esta materia, ya que si se han establecido por orden nacional que en los departamentos y municipios se implementen los planes integrales de seguridad y convivencia ciudadana, los cuales son el principal instrumento de planeación en este asunto, es preocupante que una ciudad como Cartagena que experimenta según lo analizado grandes problemas de inseguridad carezca de uno, poniendo en evidencia que los programas o estrategias establecidas en los planes de desarrollo de los últimos gobiernos estén siendo deficientes para resolver la problemática, tomando como ejemplo el Acuerdo No. 016 de 2013; periodo de gobierno en el cual el nivel de eficacia de implementación de su estrategia de seguridad ocupó un nivel medio, teniendo en cuenta lo que reportan Cartagena cómo vamos (2015) y COSED (2015), el nivel de homicidios siguió teniendo índices alarmantes que demuestran que e Plan en esta materia no fue efectivo, y su nivel de incidencia en la solución de la problemática de seguridad fue mínimo, esto se evidencia en el hecho de que en este periodo según la línea de análisis, el 2014 fue el año con mayor homicidios de los últimos 20 años; las expresiones de delito en la ciudad no pudieron ser abordadas de forma integral puesto que otros fenómenos de violencia fueron creciendo de manera importante como las manifestaciones de violencia interpersonal e intrafamiliar. Con el Plan de Desarrollo 2016-2018 se espera que se pueda lograr influir de manera más efectiva e integral en el control del delito con la ejecución del Plan Integral de Seguridad y Convivencia 
Ciudadana (PISCC, 2014) que se propone desarrollar, además se puede ver que se emplearán acciones según el Plan para atacar las nuevas manifestaciones de violencia que se están dando en la ciudad articulado con el fomento de la participación de la ciudadanía en el logro de este gran reto.

\section{REFERENCIAS BIBLIOGRÁFICAS}

Acuerdo 016 (26 de diciembre de 2013). Por medio del cual se adopta el Plan de Desarrollo del Distrito Turístico y Cultural de Cartagena de Indias - "Ahora si". Cartagena Bolivar, Colombia.

Acuerdo 06 (13 de junio de 2016). Por medio del cual se adopta el Plan de Desarrollo

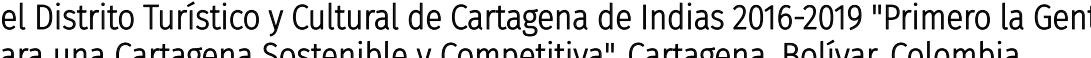
(

Alcadía Mayor de Cartagena de Indias (2016). Conclusiones y acta final de audiencia pública de rendición de cuentas a la ciudadanía de la vigencia 2016.

Candia, C. B. (2002). Seguridad ciudadana y Estado policíaco. Revista Latinoamericana Polis, 1-7.

Cartagena cómo vamos (2016). Encuesta de percepción ciudadana 2016. Obtenido de Cartagena cómo vamos: http://www.cartagenacomovamos.org/nuevo/wp-content/

Cartagena cómo vamos (2015). Informe de calidad de vida 2015. Obtenido de Cartagena 2016/07/Presentaci\%C3\%B3n-ICV-2015-Cartagena-C\%C3\%B3mo-Vamos.pdf

CELS y otros (2004). Más derechos. Más seguridad. Políticas públicas y seguridad en una sociedad democrática.

Concejo Distrital de Cartagena de Indias (2013). Acuerdo No. 016 de 2013: Por medio del Cual se adopta el Plan de Desarrollo del Distrito Turistico y Cultural de Cartagena de Indias "Ahora sí Cartagena 2013-2015".

Concejo Distrital de Cartagena de Indias D.T. y C. (2016). Acuerdo No. 006 de 2016: Plan de Desarrollo Distrital 2016-2019 Primero la Gente para una Cartagena sostenible y competitiva.

Congreso de la República de Colombia (1993). Ley 62 de 1993: Por la cual se expiden normas sobre la Policía Nacional, se crea un establecimiento público de seguridad social y bienestar para la Policía Nacional se crea la Superintendencia de Vigilancia y Seguridad Privada y se reviste de facultades. Diario Oficial No. 40.987 de agosto 12 de 1993.

Congreso de la República de Colombia (2011). Ley 1450 de 2011: Por la cual se expide el Plan Nacional de Desarrollo, 2010-2014. Diario Oficial 48102 de junio 16 de 2011. Congreso de la República de Colombia (2015). Ley 1753 de 2015: Por la cual se expide
el Plan Nacional de Desarrollo 2014-2018 "Todos por un nuevo pais". Diario Oficial No. 49.538 de 9 de junio de 2015.

Constitución Política de Colombia (1991). Colombia.

COSED (2015). Diagnóstico estadístico de delitos en Cartagena de Indias 2008-2015. Obtenido de COSED: https://www.cosed.org/portal/wp-content/uploads/2016/07/ Diagnosticofinal.pd

Duque, M. V. (Junio de 2016). Acuerdo No. 006 de 2016, Plan de Desarrollo Distrital 2016-2019: Primero la Gente para una Cartagena sostenible y competitiva. nuevo/wp-content/uploads/2016/03/comentarios-cartagena-c\%c3\%93mo-vamos-al-plan-de-desarrollo.pd
Frühling, H. (2012). La eficacia de las políticas públicas de seguridad ciudadana en America Latina y el Caribe: Cómo medirla y cómo mejorarla. Banco Interamericano de Desarrollo.

Frühling, H., Tulchin, J., Golding, H. (2005). Crimen y Violencia en América Latina: Seguridad ciudadana, Democracia y Estado.

Jaramillo, M. (2015). Los retos de la descentralización en materia de seguridad ciudadana. Revista Criminalidad, 287-299.

Ley 1450 (16 de junio de 2011). Por la cual se expide el Plan Nacional de Desarrollo, .

Ley 1753 (9 de junio de 2015). Por la cual se expide el Plan Nacional de Desarrollo

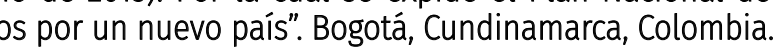

Ley 62 (12 de agosto de 1993). Por la cual se expiden normas sobre la Policía Nacional, Nacionl Cundinamarca, Colombia.

López, M. \& García, C. (2001). Políticas Públicas De Seguridad Ciudadana en el Distrito Capital. Revista de Trabajo Social, 90-103.

Marchan, C. (2004). La Seguridad Ciudadana a partir de la justicia social. En O. Jarrín Memoria del proyecto Política pública de seguridad ciudadana primera fase (pp.65-67). Ecuador: FLACSO.

Meny, Y. y Thoenig, J. (1992). Las políticas públicas. Barcelona: Editorial Ariel.

Monsalve, Y. B. (2009). Seguridad ciudadana, desempeño policial y la calidad de vida

Policía Nacional (diciembre de 2010). Políticas Públicas de Convivencia y Seguridad de Policía Nacioval de Col a Violencia, Delicus de Concia e inseguridad. Obtenido policia.edu.co/documentos/normatividad_2016/cartillas/Cartilla_3_DMS.pdf

Rivas, A. (2003). Una década de Políticas de Seguridad Ciudadana en Colombia obtenido de Georgeto/n Univerity Policul Database of the Americas: http:// politicas.pdf

Secretaría de Planeación Distrital (septiembre de 2015). Informe Técnico de Seguimiento y Evaluación del Plan de Desarrollo "Ahora si Cartagena 2013-2015" septiembre de 2015. Obtenido de Alcaldía distrital de Cartagena de indias: http: // www.cartagena.gov.co/Documentos/2016/Transparencia_y_aip/Plan_antico作 Desarrollo\%20a\%20sept\%202015.pd 